\title{
Local and global instabilities of spatially developing flows: cautionary examples
}

\author{
By L. BREVDO AND T. J. BRIDGES ${ }^{2}$ \\ ${ }^{1}$ Mathematisches Institut A, Pfaffenwaldring 57, Universität Stuttgart, \\ D-70569 Stuttgart, Germany \\ ${ }^{2}$ Department of Mathematical and Computing Sciences, University of \\ Surrey, Guildford, Surrey GU2 5XH, UK
}

In the analysis of the linear stability of basic states in fluid mechanics that are slowly varying in space, the quasi-homogeneous hypothesis is often invoked, where the stability exponents are defined locally and treated as slowly varying functions of a spatial coordinate. The set of local stability exponents is then used to predict the global perturbation dynamics and an implicit hypothesis is that the local analysis provides at least a conservative estimate of the global stability properties of the flow. In this paper cautionary examples are presented that demonstrate a contradiction between the results of the local and global analyses. For example, a local analysis may predict stability everywhere even when the exact PDE with non-constant coefficients is ill-posed, demonstrating that global stability exponents are not, in general, bounded by the maximal local stability exponents. A key observation in this paper is the importance of distinguishing between the discrete spectrum and the continuous spectrum when comparing global and local stability exponents. This distinction is particularly significant for spatially periodic flows where, for the global flow, only the continuous spectrum is present and, hence, instability arises always in the absence of discrete spectra. New exact definitions for global absolute and convective instabilities are also given for a class of spatially periodic basic states and applied to an example based on the complex Ginzburg-Landau equation. The consequences of this example, and of the argument involved for basic states that are slowly varying in space but non-periodic, and for some problems in fluid mechanics are also presented.

\section{Introduction}

In the treatment of the linear stability of fluid flows, where the basic flow varies slowly in the streamwise direction, the quasi-homogeneous hypothesis, or parallel flow assumption, in which the linear stability exponent is taken to depend on the streamwise coordinate, is often invoked as an approximation to the global stability problem. The fundamental premise is that, in a stationary flow whose properties vary slowly with a spatial coordinate, say $x$, the global linear stability growth rates are well approximated by the linear stability growth rates of a union of fictitious parallel flows. In this setting the term 'slowly varying' means that the spatial variation scale $L_{0}$ of the flow is significantly greater than the characteristic wavelength $\lambda_{0}$ of the most unstable (or least stable) monochromatic disturbances of the corresponding parallel flow. In such a case, it is argued, the behaviour of the dominant monochromatic waves

Proc. R. Soc. Lond. A (1997) 453, 1345-1364

Printed in Great Britain 
in the neighbourhood $\left\{x: x_{1}<x<x_{2}\right\}$ of the point $x_{0}$, with $\lambda_{0} \ll x_{2}-x_{1} \ll L_{0}$, is influenced only by the local flow properties, which by the hypothesis are constant to within a negligible quantity, in this neighbourhood. Since any realistic disturbance is a linear superposition of monochromatic waves, the global-accounting for all $x_{0} \in \mathbb{R}$ - linear dynamics of localized perturbations (wave packets) are therefore reduced, in the quasi-parallel approach, to the combined effect of a union of the local dynamics of the corresponding fictitious parallel flows.

By definition, the global linear stability problem accounts for the total $x$-dependent flowfield (Huerre \& Monkewitz 1990, hereafter HM). When the basic state depends only on $x$, a global normal mode is defined by the ansatz $Z(x, t)=\hat{Z}(x) \mathrm{e}^{-\mathrm{i} \omega_{\mathrm{G}} t}$, where $\omega_{\mathrm{G}}$, the global stability exponent, is independent of $x$ and $\hat{Z}(x)$ must satisfy a boundary-value problem (BVP) on the real line. But what boundary conditions should be imposed on $\hat{Z}(x)$ as $x \rightarrow \pm \infty$ ? In $\S 3.2$ of HM, a global normal mode is required to satisfy a decay condition as $x \rightarrow \pm \infty$. Then, once the BVP is specified, a flow is called globally stable if $\operatorname{Im}\left(\omega_{\mathrm{G}}\right) \leqslant 0$ for all global modes. If, for at least one global mode, $\operatorname{Im}\left(\omega_{\mathrm{G}}\right)>0$, the flow is globally unstable. However, we shall see that, at least from the perspective of treating spatially periodic flows, it is necessary to expand this definition to require only boundedness of $\hat{Z}(x)$ as $x \rightarrow \pm \infty$, since normal modes, for spatially periodic states, are in general quasi-periodic in space and therefore do not decay as $x \rightarrow \pm \infty$. On the other hand, we do not attempt to give here a definition of global instability which would cover all possible physically relevant spatially varying flows.

Contrary to the global definition, the definition of local instability is clear and generally accepted: for a basic state that depends on $x$, regardless of whether the variation is slow, a local instability treatment amounts to an analysis of the linear stability problem with frozen coefficients, resulting in a set of local, $x$-dependent, stability exponents. For slowly spatially varying flows, an implicit hypothesis is that the collection of local stability exponents can be used to predict features of the global stability problem or to provide conservative estimates of the global exponents.

Because of the difficulty with studying the exact global stability problem, considerable effort has been devoted to constructing approximate theories for global instability, principally through the use of WKBJ theory. Inconsistencies may arise, however, when approximate definitions for global instability are introduced. In this paper, an exact analysis of the the global stability problem for a class of spatially periodic basic states is used to construct counterexamples to the quasi-homogeneous approximation for the linear stability of slowly varying flows. In the first example, presented in $\S 2$, the global exponent is not bounded from above by the maximum local growth rate. In the example, the basic state is everywhere locally stable and so the upper bound for the set of local growth rates is zero, whereas the global problem is unstable in the most extreme way, namely it has unbounded growth rates. A second example is treated in $\S 4$. This example is based on the stability problem for exact travelling wave solutions of the complex Ginzburg-Landau equation, a model equation for a class of near-critical hydrodynamic stability problems. The analysis shows that, even when the $x$ dependence of the basic state is very slow, the quasi-homogeneous hypothesis or parallel flow assumption gives results that directly contradict the exact global linear stability results. Because of the special nature of spatially periodic flows, where an exact analysis is possible using spatial Floquet theory, it is possible to give a definition for global absolute and convective instabilities

Proc. R. Soc. Lond. A (1997) 
for such flows based on first principles. In $\S 4$, the the global absolute and convective instabilities are compared with local absolute and convective instabilities.

Among phenomena governed by ODEs with periodic coefficients, there is a classic problem in mechanics that illustrates in a simple setting the dramatic difference between a local stability exponent and a global stability exponent. A fundamental problem in classical mechanics is the stabilization of an inverted pendulum by periodically forcing its pivot point. The linear stability problem reduces to the Mathieu equation for the vertical position $y(t)$ of the pivot point

$$
y_{t t}+(\mu+\varepsilon \cos t) y=0, \quad \mu<0
$$

(cf. Acheson 1993). Via the quasi-static hypothesis the stability exponents for (1.1), for any fixed $t_{0}$, are easily computed to be

$$
\lambda\left(t_{0}\right)= \pm\left(|\mu|-\varepsilon \cos t_{0}\right)^{1 / 2} .
$$

If $|\varepsilon|$ is sufficiently large, there will be values of $t$, for which $|\mu|>\varepsilon \cos t$ and $|\mu|<$ $\varepsilon \cos t$, resulting in open $t_{0}$ regions of local instability and stability. However, the global stability properties of this problem are well known (cf. Acheson 1993, figure 2). The periodically forced inverted pendulum is stable (unstable) if and only if the globally defined Floquet exponents are stable (unstable). The distinction between the global and local values of the stability exponents is even more dramatic when the forcing function is taken to be piecewise constant (a periodic square wave):

$$
y_{t t}+[\mu+\varepsilon \operatorname{sgn}(\cos t)] y=0, \quad \mu<0, \quad \varepsilon>0 .
$$

For this problem, there exist values of $(\mu, \varepsilon)$, with $|\mu|<\varepsilon$, such that the system is globally stable (cf. Levi \& Weckesser 1995), whereas the local stability exponent

$$
\lambda\left(t_{0}\right)= \pm\left[|\mu|-\varepsilon \operatorname{sgn}\left(\cos t_{0}\right)\right]^{1 / 2}
$$

is stable for half the period: $t_{0} \in\left(-\frac{1}{2} \pi, \frac{1}{2} \pi\right)$ and unstable for the other half. Thus, the local $t_{0}$-dependent values of the stability exponents can be significantly misleading with respect to the global stability. Although the $t_{0}$-dependent exponents, such as in (1.2) or (1.4), can provide some information about the system, it is clear, mathematically, that the stability of the system is determined by the globally defined Floquet exponents only. The theoretical predictions of the linear stability of the inverted pendulum have also been supported by experiments (cf. Acheson \& Mullin 1993).

In fluid mechanics, in particular, for time-varying but spatially homogeneous flows, the inherent contradiction between local and global instabilities has been recognized previously (cf. von Kerczek \& Davis 1974; Hall 1978). For example, when the basic state is time periodic, such as in the case of the oscillating Stokes layer, the instantaneous velocity profiles are highly inflectional and inviscidly locally unstable even for Reynolds numbers at which the flow is globally stable. While the local properties of a particular oscillating flow may be of some significance for other questions, from the point of view of the linear stability, the oscillating flow is linearly stable (unstable) if and only if the globally defined Floquet exponents are stable (unstable).

The analysis of spatially slowly varying flows in fluid mechanics is important for the investigation of the stability and control of boundary and mixing layers, jets and wakes. For such investigations, the parallel flow or weakly parallel assumption is often invoked (cf. Drazin \& Reid 1981; Huerre \& Monkewitz 1990 and the references therein). With regard to the streamwise variation of such flows, it is important to note that there are two distinct questions. First, the solutions for mixing and boundary

Proc. R. Soc. Lond. A (1997) 
layers, jets and wakes, used in linear stability investigations, are not in general exact, but are asymptotic, solutions of the Navier-Stokes equations. Therefore, in order to formulate, in a mathematically consistent manner, the linear stability problem one must first balance the asymptotics of the basic state with an asymptotic formulation of the linear stability problem. While there are many outstanding questions with regard to the asymptotic aspects of the stability of shear flows, the most successful analysis in this direction has been with the multi-deck framework (cf. Smith (1979), where this framework was first proposed for boundary layer instability and Stewart \& Smith (1987) for further results and references). However, there is a second question that already appears even when the slowly spatially varying basic state is known exactly and it is precisely this question that we address in this paper.

For a class of basic states, namely basic states whose $x$ dependence is spatially periodic, an exact characterization of global instability as well as the global absoluteconvective instability dichotomy, can be constructed (cf. Brevdo \& Bridges 1996, hereafter $\mathrm{BB}$ ). Therefore, the class of spatially periodic $x$-dependent basic states provides an interesting setting, where approximate definitions of global instability can be tested. In BB, the exact characterization is constructed for global instability using a spatial Floquet decomposition, leading to an analytical theory for treating wave packets in such flows. The theory was applied to linear disturbances of periodic travelling wave solutions of the Ginzburg-Landau equation and used to formulate the classification of secondary instabilities of shear flows. This theory makes it possible to study exactly the global stability problem and compare the results with those based on the parallel flow assumption in a mathematically precise way for an arbitrarily large spatial variation scale. The theory in BB shows that the linear dynamics of any spatially periodic flow are essentially global regardless of the spatial variation rate and the period of the flow. Consequently, an application of the parallel flow assumption to a spatially periodic flow can be justified only if it is a priori known that the local velocity profiles possess the same dynamics as the flow on the whole. It is the interplay between the local space dependent stability exponents and the globally defined spatial Floquet exponents, coupled with the fact that the spectral problem arising from the linearization about a spatially periodic state has only a continuous spectrum, that forms the backbone of the argument of this paper.

The paper is organized as follows. In $\S 2$, we address inconsistencies that may arise while comparing local and global stability exponents. An example is presented that shows that local growth rates do not in general bound global growth rates. This is due to the nature of the spectrum of the exact operator in the stability equation and the continuous spectrum of the linearized operator. Both spectra play a central role in the global-local stability treatment of spatially periodic flows. In $\S 3$, a short review of the theory in BB is given and the question of the streamwise spatial variability of the asymptotics of wave packets in periodic media is discussed. In $\S 4$, we treat the complex Ginzburg-Landau (CGL) equation along a curve in the parameter space where the basic periodic states are all unstable except for the spatially homogeneous state which is neutrally stable. The exact results for the global instability exponents are compared to results obtained using the locally homogeneous assumption. In $\S 5$, the consequences of the dichotomy between locally defined stability exponents and a global definition of stability for problems in fluid mechanics are discussed, with additional remarks concerning the applicability of the technique of the parallel flow assumption and the WKBJ technique to studying instabilities in an arbitrary slowly

Proc. R. Soc. Lond. A (1997) 
spatially varying flow that can be modelled as periodic in the homogeneous spatial direction.

\section{Comparing local and global stability exponents}

A central analytical result in the perturbation stability theory of spatially developing flows that relates local and global instabilities is the inequality

$$
\operatorname{Im}\left(\omega_{\mathrm{G}}\right) \leqslant \operatorname{Im}\left[\omega_{0}\left(X_{\mathrm{s}}\right)\right] \leqslant \sup _{X \in \mathbb{R}} \operatorname{Im}\left[\omega_{0}(X)\right],
$$

where $\omega_{\mathrm{G}}$ is the global stability exponent, $\left\{\omega_{0}(X): X \in \mathbb{R}\right\}$ is the set of local stability exponents and $X_{\mathrm{s}}$ is a saddle point of the function $\omega_{0}(X)$ in the complex $X$ plane that lies closest to the real $X$ axis (HM, p. 494). A proof of this inequality for a class of Ginzburg-Landau type equations has been given, in the WKBJ framework, in Chomaz et al. (1991) (hereafter CHR). When valid, this inequality predicts that the growth rate of a globally unstable mode cannot exceed the maximum local growth rate. A corollary of (2.1) is that an everywhere locally stable basic state cannot be globally unstable.

In this section, a counterexample to the inequality (2.1) is presented in which the localized equation is everywhere stable while the global analysis predicts instability in the most extreme way - the PDE is ill-posed. Moreover, this contradiction is maintained no matter how slowly the non-constant coefficients vary in space. The conclusion is that the inequality (2.1) is false in general. However, it clearly holds in some cases. So, after treating the counterexample, the proof of the inequality (2.1) given in CHR will be re-examined and its validity for other equations, including the Navier-Stokes equations, will be assessed.

We consider the following PDE:

$$
u_{t}=\boldsymbol{A}(x) u_{x x}, \quad x \in \mathbb{R}, \quad t \geqslant 0, \quad u \in \mathbb{C}^{2},
$$

where $u=u(x, t)$,

$$
\mathbf{A}(\mathbf{x})=\mathrm{i} \boldsymbol{G}(\mathbf{x})^{\mathrm{T}} \boldsymbol{F} \boldsymbol{G}(\mathbf{x}), \quad \boldsymbol{G}(\mathbf{x})=\left(\begin{array}{cc}
\cos \varepsilon x & -\sin \varepsilon x \\
\sin \varepsilon x & \cos \varepsilon x
\end{array}\right)
$$

and

$$
\boldsymbol{F}=\left(\begin{array}{ll}
a & c \\
0 & b
\end{array}\right) \quad \text { with } a, b, c \in \mathbb{R}, \quad a \neq b, \quad a \cdot b \cdot c \neq 0 .
$$

The $x$ dependent matrix $\boldsymbol{A}(x)$ is $L$ periodic with $L=2 \pi / \varepsilon$. This PDE is a modification of an example due to Kreiss \& Lorenz (1989, p. 77).

A local, or frozen coefficient, analysis of (2.2) proceeds as follows. Let $\boldsymbol{G}_{0}=\boldsymbol{G}\left(x_{0}\right)$, where $x_{0}$ is considered fixed. Then, with $\boldsymbol{A}_{0}=\boldsymbol{A}\left(x_{0}\right)$, equation (2.2), that is $u_{t}=$ $\boldsymbol{A}_{0} u_{x x}$, is a PDE with constant coefficients. The transformation $w(x, t)=\boldsymbol{S G}_{0} u(x, t)$ reduces it to

$$
w_{t}=\mathrm{i}\left(\begin{array}{cc}
a & 0 \\
0 & b
\end{array}\right) w_{x x},
$$

where the constant matrix $\boldsymbol{S}$ is chosen to satisfy $\boldsymbol{S} \boldsymbol{F} \boldsymbol{S}^{-1}=\operatorname{diag}[a, b]$ (see (2.4)). A normal mode ansatz, $w(x, t)=\hat{w} \mathrm{e}^{\mathrm{i}\left(k x-\omega_{0} t\right)}$, applied to (2.5) leads to the localized 
dispersion relation

$$
D\left(\omega_{0}, k\right)=\operatorname{det}\left[\mathrm{i}\left(\begin{array}{ll}
a & 0 \\
0 & b
\end{array}\right) k^{2}-\mathrm{i} \omega_{0} \boldsymbol{I}_{2}\right]=-\left(\omega_{0}-a k^{2}\right)\left(\omega_{0}-b k^{2}\right),
$$

where $\boldsymbol{I}_{2}$ is the $2 \times 2$ identity matrix. It is evident that, for any fixed $x_{0} \in \mathbb{R}$, the local analysis predicts stability for any $a, b \in \mathbb{R}$ and $\sup _{x_{0} \in \mathbb{R}} \operatorname{Im}\left[\omega_{0}\left(x_{0}\right)\right]=0$.

The coefficients in (2.2) are spatially periodic and the global stability exponents for the PDE can be completely analysed using a Floquet decomposition. Because of the form of $\boldsymbol{A}(x)$, the PDE can be transformed into a PDE with constant coefficients. Substitution of $v(x, t)=\boldsymbol{G}(x) u(x, t)$ into (2.2) results in the following PDE for $v$ :

$$
v_{t}=\mathrm{i} \boldsymbol{F} v_{x x}-2 \mathrm{i} \varepsilon \boldsymbol{F} \boldsymbol{J} v_{x}-\mathrm{i} \varepsilon^{2} \boldsymbol{F} v, \quad \text { where } \boldsymbol{J}=\left(\begin{array}{cc}
0 & -1 \\
1 & 0
\end{array}\right) .
$$

A normal mode ansatz, $v(x, t)=\hat{v} \mathrm{e}^{\mathrm{i}\left(k x-\omega_{\mathrm{G}} t\right)}$, leads to the global dispersion relation function

$$
D\left(\omega_{\mathrm{G}}, k\right)=\operatorname{det}\left[\boldsymbol{P}(k)-\mathrm{i} \omega_{\mathrm{G}} \boldsymbol{I}_{2}\right]=-\omega_{\mathrm{G}}^{2}-\mathrm{i} \omega_{\mathrm{G}} \operatorname{tr}[\boldsymbol{P}(k)]+\operatorname{det}[\boldsymbol{P}(k)],
$$

where

$$
\boldsymbol{P}(k)=\mathrm{i} \boldsymbol{F}\left[\left(k^{2}+\varepsilon^{2}\right) \boldsymbol{I}_{2}+2 \mathrm{i} \varepsilon k \boldsymbol{J}\right] .
$$

The dispersion relation function is quadratic in $\omega_{\mathrm{G}}$ with two complex roots, denoted $\omega_{\mathrm{G}}^{(1)}(k)$ and $\omega_{\mathrm{G}}^{(2)}(k)$. The roots satisfy

$$
\operatorname{Im}\left[\omega_{\mathrm{G}}^{(1)}(k)+\omega_{\mathrm{G}}^{(2)}(k)\right]=\operatorname{Re}\{\operatorname{tr}[\boldsymbol{P}(k)]\}=2 \varepsilon k c .
$$

Hence, since $c \neq 0$, the imaginary part of one of the $\operatorname{roots} \operatorname{Im}\left[\omega_{\mathrm{G}}^{(1)}(k)\right], \operatorname{Im}\left[\omega_{\mathrm{G}}^{(2)}(k)\right]$, say $\operatorname{Im}\left[\omega_{\mathrm{G}}^{(1)}(k)\right]$, is positive and unbounded in the limit $k \rightarrow \operatorname{sgn}(c) \infty$; that is, there is no upper bound for the growth rates of the global normal modes, for real wave numbers $k$. Moreover, this is true for any fixed $\varepsilon$. Since $L_{0}=2 \pi / \varepsilon$ is a spatial variation scale of the coefficients of (2.2), this PDE with non-constant coefficients is not only unstable but also ill-posed as an evolution equation, for arbitrarily large $L_{0}$. The inequality (2.1) in this case is violated in the strongest possible way: the maximum localized growth rate $\sup _{x_{0} \in \mathbb{R}} \operatorname{Im}\left[\omega_{0}\left(x_{0}\right)\right]$ is zero, whereas the global growth rate $\operatorname{Im}\left[\omega_{\mathrm{G}}^{(1)}(k)\right]$ takes every possible positive real value with no upper bound.

The concept of localization of PDEs with non-constant coefficients, when the coefficients are frozen, is discussed for general classes of PDEs in Kreiss \& Lorenz (1989). In particular, they present the following example:

$$
u_{t}=\mathrm{i} p(x) u_{x x}+\mathrm{i} p_{x}(x) u_{x}, \quad x \in \mathbb{R}, \quad t \geqslant 0,
$$

where $u(x, t)$ is a complex-valued scalar function and $p(x)$ is a real smooth periodic function satisfying $p(x) \geqslant p_{0}>0$. In this example, compared to (2.2), the situation is reversed, namely the exact $\mathrm{PDE}$ with variable coefficients is well-posed, whereas the PDE with frozen coefficients is ill-posed; that is, the local exponents have unbounded growth rates but the system is globally stable.

The counterexample (2.2) demonstrates that the inequality (2.1) is not valid in general. However, it does hold for certain classes of PDEs under suitable hypotheses. In CHR, a proof of (2.1) is given for a class of Ginzburg-Landau (GL) type PDEs. In order to outline the premises assuring the validity of (2.1), we present the main points

Proc. R. Soc. Lond. A (1997) 
of the proof. The linearized GL equation is reduced to the following boundary-value spectral problem:

$$
\varepsilon^{2} \omega_{k k} \phi_{X X}+2\left[\omega_{\mathrm{G}}-\tilde{\omega}_{0}(X)\right] \phi=0, \quad X \in \mathbb{R},
$$

cf. equation (A1) in CHR, where $\omega_{\mathrm{G}}$ is the global stability exponent and $\tilde{\omega}_{0}(X)$ represents the $X$-dependent basic state. It is assumed that $\omega_{\mathrm{G}}$ belongs to the eigenvalue spectrum of the operator

$$
\boldsymbol{L}(X) \phi=\left[-\frac{1}{2} \varepsilon^{2} \omega_{k k} \frac{\mathrm{d}^{2}}{\mathrm{~d} X^{2}}+\tilde{\omega}_{0}(X)\right] \phi, \quad X \in \mathbb{R},
$$

with an eigenfunction $\phi(X)$ satisfying

$$
\|\phi\|_{2}^{2}=\int_{-\infty}^{\infty}|\phi(X)|^{2} \mathrm{~d} X<\infty \quad \text { and } \quad\left\|\phi_{X}\right\|_{2}^{2}=\int_{-\infty}^{\infty}\left|\phi_{X}(X)\right|^{2} \mathrm{~d} X<\infty .
$$

This assumption implies that, in the definition of global stability, the global normal modes are

$$
\phi(X) \mathrm{e}^{-\mathrm{i} \omega_{\mathrm{G}} t}, \quad \text { with } \phi \in L_{2}(\mathbb{R}) .
$$

Multiplication of $(2.12)$ by $\phi^{*}(X)$, where the superscript $*$ denotes complex conjugation, integration and taking the imaginary part leads to

$$
\operatorname{Im}\left(\omega_{\mathrm{G}}\right)\|\phi\|_{2}^{2}=\int_{-\infty}^{\infty} \operatorname{Im}\left[\tilde{\omega}_{0}(X)\right]|\phi(X)|^{2} \mathrm{~d} X+\frac{1}{2} \varepsilon^{2} \operatorname{Im}\left(\omega_{k k}\right)\left\|\phi_{X}\right\|_{2}^{2} .
$$

The assumption $\operatorname{Im}\left(\omega_{k k}\right)<0$ in CHR finally reduces (2.16) to

$$
\operatorname{Im}\left(\omega_{\mathrm{G}}\right) \leqslant \sup _{X \in \mathbb{R}} \operatorname{Im}\left[\tilde{\omega}_{0}(X)\right] .
$$

We note that the two sides of (2.17) have different meanings. According to the definition of $\omega_{\mathrm{G}}$, the left-hand side corresponds to the eigenvalue spectrum of the operator $\boldsymbol{L}(X)$ given in $(2.13)$ in the space $L_{2}(\mathbb{R})$. The right-hand side is related to the union of the continuous spectra of the localized, frozen coefficient, operators

$$
\boldsymbol{L}\left(X_{0}\right) \phi=\left[-\frac{1}{2} \varepsilon^{2} \omega_{k k} \frac{\mathrm{d}^{2}}{\mathrm{~d} X^{2}}+\tilde{\omega}_{0}\left(X_{0}\right)\right] \phi, \quad \text { real } X_{0} \text { is fixed },
$$

in the same space. Indeed, the eigenvalue spectrum of $\boldsymbol{L}\left(X_{0}\right)$ in $L_{2}(\mathbb{R})$ is empty, for every fixed $X_{0}$. Its continuous spectrum $C\left(X_{0}\right)$ consists of a semi-line

$$
C\left(X_{0}\right)=\left\{\lambda \mid \lambda \in \mathbb{C}, \lambda=\tilde{\omega}_{0}\left(X_{0}\right)+a^{2}\left(\frac{1}{2}\right) \epsilon^{2} \omega_{k k}, a \in \mathbb{R}\right\} .
$$

For a fixed $X_{0}$, it holds that $\sup \left\{\operatorname{Im}(\lambda) \mid \lambda \in C\left(X_{0}\right)\right\}=\operatorname{Im}\left[\tilde{\omega}_{0}\left(X_{0}\right)\right]$, because $\operatorname{Im}\left(\omega_{k k}\right)<0$. The supremum is attained for $a=0$. Therefore, a conclusion expressed in the inequality $(2.17)$ is that the maximum value of the imaginary part of the eigenvalue spectrum of the global eigenvalue problem for the operator $\boldsymbol{L}(X)$ given in (2.13) is not greater than the maximum imaginary part of an element in the union of the continuous spectra of all localized problems, i.e. those for the operators $\boldsymbol{L}\left(X_{0}\right)$ in $(2.18)$.

An explicit example illustrating the distinction between the left- and right-hand sides of (2.17) is the following spectral problem:

$$
\mathrm{i} \phi_{x x}+\mathrm{i}\left[a+b-b \tanh ^{2}(\varepsilon x)\right] \phi=\lambda \phi, \quad a \in \mathbb{R}, \quad x \in \mathbb{R} .
$$

Proc. R. Soc. Lond. A (1997) 
The spectrum of $(2.19)$ in $L_{2}(\mathbb{R})$, with $\lambda$ as spectral parameter, can be determined exactly by transforming this equation into an associated Legendre equation (cf. Drazin \& Johnson 1989, §3.2). The eigenvalue spectrum is non-empty if $b=\varepsilon^{2} N(N+1)$, for a natural number $N$. Then there are $N$ discrete eigenfunctions with eigenvalues $\lambda_{n}=\mathrm{i}\left(a+\varepsilon^{2} n^{2}\right)$, for $n=1,2, \ldots, N$. The continuous spectrum of the operator in (2.19) consists of the set of $\lambda=\mathrm{i} \mu, \mu \in \mathbb{R}$, such that $\mu \leqslant a$. On the other hand, each localized operator obtained by freezing $x$ in the coefficient of $\phi$ in the operator on the left-hand side of (2.19) has only a continuous spectrum which (by varying $x$ for all $\mathbb{R})$ covers the imaginary $\lambda$ axis from $-\mathrm{i} \infty$ to the point $\mathrm{i}\left[a+\varepsilon^{2} N(N+1)\right]$, when $b=\varepsilon^{2} N(N+1)$. We have

$$
\max _{n}\left\{\operatorname{Im}\left(\lambda_{n}\right)\right\}=a+\varepsilon^{2} N^{2}<a+\varepsilon^{2} N(N+1) .
$$

This illustrates that, in the case when the eigenvalue spectrum of the exact equation is non-empty, the imaginary part of any eigenvalue in this spectrum is not greater than the maximum value of the imaginary part of an element in the union of the continuous spectra of the localized spectral problems. Hence, the conclusion expressed in (2.17) is certainly valid for (2.13) and (2.19), but as we shall see in a sequel it does not hold in general.

As a matter of fact, with careful attention to the underlying assumptions, the above argument can be extended to the eigenvalue spectrum, in $L_{2}(\mathbb{R})$, of the Navier-Stokes equations linearized about a spatially varying state. Such an analysis is based on the Reynolds-Orr stability theory. Let $\boldsymbol{u}=(u, v, w)$ be a velocity field in a cylinder $\{\boldsymbol{x}=(x, y, z): x \in \mathbb{R},(y, z) \in \mathcal{C}\}$, where $\mathcal{C}$ is a bounded set. Let $(\boldsymbol{U}(\boldsymbol{x}), P(\boldsymbol{x}))$ be a velocity and pressure field satisfying the steady Navier-Stokes equations for some Reynolds number $R$ :

$$
\boldsymbol{D}(\boldsymbol{U}) \boldsymbol{U}+\nabla P=\frac{1}{R} \Delta \boldsymbol{U}, \quad \nabla \cdot \boldsymbol{U}=0
$$

where

$$
\boldsymbol{D}(\boldsymbol{U})=\left(\begin{array}{ccc}
U_{x} & U_{y} & U_{z} \\
V_{x} & V_{y} & V_{z} \\
W_{x} & W_{y} & W_{z}
\end{array}\right) .
$$

The time-dependent Navier-Stokes equations, linearized about $(\boldsymbol{U}, P)$ read

$$
\boldsymbol{u}_{t}+\boldsymbol{D}(\boldsymbol{U}) \boldsymbol{u}+\boldsymbol{D}(\boldsymbol{u}) \boldsymbol{U}+\nabla p-\frac{1}{R} \Delta \boldsymbol{u}=0, \quad \nabla \cdot \boldsymbol{u}=0,
$$

with $(\boldsymbol{u}, p)$ being the perturbation field and $\boldsymbol{u}=0$ on the boundary of the cylinder. If we assume that $\boldsymbol{u}$ is square integrable in the cylinder $\mathcal{C} \times \mathbb{R}$, then the usual ReynoldsOrr energy argument results in

$$
\frac{\partial}{\partial t} \int_{\mathcal{C} \times \mathbb{R}} \boldsymbol{u} \cdot \boldsymbol{u}+2 \int_{\mathcal{C} \times \mathbb{R}} \boldsymbol{u} \cdot \boldsymbol{D}(\boldsymbol{U}) \boldsymbol{u}+\frac{2}{R} \int_{\mathcal{C} \times \mathbb{R}} \operatorname{tr}\left[\boldsymbol{D}(\boldsymbol{u})^{\mathrm{T}} \boldsymbol{D}(\boldsymbol{u})\right]=0
$$

(cf. Serrin 1959, equation (4)). Consequently,

$$
\frac{\partial}{\partial t} \int_{\mathcal{C} \times \mathbb{R}} \boldsymbol{u} \cdot \boldsymbol{u} \leqslant-2 \int_{\mathcal{C} \times \mathbb{R}} \boldsymbol{u} \cdot \boldsymbol{D}(\boldsymbol{U}) \boldsymbol{u} .
$$

Therefore, the temporal growth rates of the modes associated with the eigenvalue Proc. R. Soc. Lond. A (1997) 
spectrum are bounded from above by

$$
\max \left\{-2 \int_{\mathcal{C} \times \mathbb{R}} \boldsymbol{u} \cdot \boldsymbol{D}(\boldsymbol{U}) \boldsymbol{u} / \int_{\mathcal{C} \times \mathbb{R}} \boldsymbol{u} \cdot \boldsymbol{u}\right\},
$$

which can be related to the (real) eigenvalues of the deformation tensor: $\boldsymbol{D}(\boldsymbol{U})+$ $\boldsymbol{D}(\boldsymbol{U})^{\mathrm{T}}$ (see Serrin (1959) for an analysis of this maximization problem). The upper bound for the eigenvalue spectrum can then be compared with the continuous spectrum of the localization of (2.22).

The above argument for the Navier-Stokes equations and for (2.12), leading to (2.17) which is a part of (2.1), is for the eigenvalue spectrum. However, it is important to emphasize that this argument does not account for the existence of unstable normal modes, of the exact PDE with non-constant coefficients, whose frequencies belong to the continuous spectrum of the exact PDE. Such normal modes are not necessarily square integrable on the real line and, when they are not, the argument leading to the inequality (2.17) is no longer valid. In such a situation, the inequality (2.1) may be violated. This is precisely the case for the example (2.2): the unstable states there correspond to normal modes associated with the continuous spectrum. These normal modes are quasi-periodic in the spatial coordinate and, therefore, not square integrable on $\mathbb{R}$. More importantly, the example (2.2) is not an isolated case. Indeed, for a class of spatially developing flows, namely two-dimensional basic states periodic in one homogeneous spatial direction, stability is determined in terms of the normal mode solutions associated with the continuous spectrum of the exact PDE - the discrete spectrum is empty. A proof that the eigenvalue spectrum of an ODE with periodic coefficients is empty and its spectrum consists entirely of the continuous spectrum is given in Dunford \& Schwartz (1963, p. 1486) and Eastham $(1973, \S 5.3)$. For general PDEs with periodic coefficients, the spectral theory is more delicate (cf. Eastham 1973, § 6.10; Kuchment 1993, p. 169).

In $\S 3$, the linear stability analysis, including the classification of absolute and convective instabilities, of spatially periodic states will be outlined and in $\S 4$, an example of such a state will be given, where the presence of the continuous spectrum of the exact global stability equation causes a violation of the inequality (2.1).

\section{Normal modes and wave packets in periodic flows}

In a theory developed in BB, it was shown that an initial boundary-value problem for localized linear disturbances in periodic media or flows, including those governed by the discretized Navier-Stokes equations, can be formulated in the following way:

$$
\left.\begin{array}{c}
M Z_{t}+Z_{x}=\boldsymbol{A}(x) Z+G(x, t), \quad x \in \mathbb{R}, \quad t>0, \\
Z(x, t)_{\mid t=0}=Z_{0}(x), \quad Z_{0}(x) \in C_{0}^{\infty}\left(\mathbb{R}, \mathbb{R}^{N}\right), \\
\lim _{x \rightarrow \pm \infty}\|Z(x, t)\|=0, \quad \text { for each } t>0 .
\end{array}\right\}
$$

Here $\boldsymbol{M}$ is a constant $N \times N$ matrix, $\boldsymbol{A}(x)$ is an $L$-periodic $N \times N$ matrix and $G(x, t) \in C_{0}^{\infty}\left(\mathbb{R} \times \mathbb{R}, \mathbb{R}^{N}\right)$, where $C_{0}^{\infty}$ indicates $C^{\infty}$ functions with compact support. The problem is formally analytically solved by operating on (3.1) with a Laplace transform, applying a Floquet transformation to the resulting ordinary differential equation with periodic coefficients and treating the transformed equation with constant coefficients by means of a Fourier transform. The solution of the initial

Proc. R. Soc. Lond. A (1997) 
boundary-value problem (3.1) is then formally expressed as

$$
Z(x, t)=\frac{1}{4 \pi^{2}} \int_{\mathrm{i} \sigma-\infty}^{\mathrm{i} \sigma+\infty} \boldsymbol{Q}(x, \omega) \int_{-\infty}^{\infty}\left[\mathrm{i} k \boldsymbol{I}_{N}-\boldsymbol{B}(\omega)\right]^{-1} T(k, \omega) \mathrm{e}^{\mathrm{i}(k x-\omega t)} \mathrm{d} k \mathrm{~d} \omega .
$$

Here the matrix $\boldsymbol{B}(\omega)$ is defined using the monodromy operator $\boldsymbol{\Phi}(L, \omega)$ :

$$
\boldsymbol{\Phi}(L, \omega)=\mathrm{e}^{\boldsymbol{B}(\omega) L},
$$

where the $N \times N$ matrix $\boldsymbol{\Phi}(x, \omega)$ satisfies the matrix initial-value problem

$$
\boldsymbol{\Phi}_{x}(x, \omega)=[\boldsymbol{A}(x)+\mathrm{i} \omega \boldsymbol{M}] \boldsymbol{\Phi}(x, \omega), \quad \boldsymbol{\Phi}(0, \omega)=\boldsymbol{I}_{N},
$$

with $\boldsymbol{I}_{N}$ being the $N \times N$ identity matrix and the matrix function

$$
\boldsymbol{Q}(x, \omega)=\boldsymbol{\Phi}(x, \omega) \mathrm{e}^{-\boldsymbol{B}(\omega) x}
$$

is $L$ periodic in $x$ (cf. BB for details). For the purpose of presentation we assume that (i) the matrix $\boldsymbol{A}(x)$ is analytic in $x$ and (ii) the matrix $\boldsymbol{B}(\omega)$ is analytic in $\omega$. The first assumption means that the exact periodic solution of the original nonlinear equations is an analytic function of the spatial coordinate $x$, which is quite realistic. The second one is valid for the solutions of the Ginzburg-Landau equation and is a reasonable conjecture for the general case. Under the above hypothesis, both $\boldsymbol{Q}(x, \omega)$ and $\boldsymbol{\Phi}(x, \omega)$ are analytic functions of both $x$ and $\omega$. The vector function $T(k, \omega)$ in (3.2) depends on the initial conditions and, therefore, can be viewed in a certain sense as arbitrary. The functions $\boldsymbol{B}(\omega)$ and $\boldsymbol{Q}(x, \omega)$ depend solely on the operator in the equation in (3.1). Consequently, the model dependent properties of solution (3.2) depend only on the functions $\boldsymbol{B}(\omega)$ and $\boldsymbol{Q}(x, \omega)$.

The normal modes of the periodic basic state have the form

$$
Z(x, t)=\boldsymbol{Q}(x, \omega) h \mathrm{e}^{\mathrm{i}(k x-\omega t)},
$$

where $h \in \mathbb{C}^{N}$ is a constant vector and the wave number $k$ and the frequency $\omega$ satisfy the dispersion relation

$$
D(k, \omega) \equiv \operatorname{det}\left[\mathrm{i} k \boldsymbol{I}_{N}-\boldsymbol{B}(\omega)\right]=0 .
$$

An immediate stunning consequence of the representations (3.6), (3.7) is that the wave numbers and the frequencies of the normal modes do not depend on $x$ regardless of the local $x$-dependent properties of the matrix function $\boldsymbol{A}(x)$ representing the periodic basic state. This means that the parallel flow assumption can only then be applied for treating instabilities in a periodic flow when the dispersion relation of a parallel flow for every fixed $x$ approximates (3.7). As we shall see in the next section this is not the case at all for exact periodic solutions of the Ginzburg-Landau equation, but we can take our theoretical argument even further by considering the time asymptotics of solution (3.2) in a frame of reference moving at speed $V$. The dominant contribution to these asymptotics, along the ray $x=x_{1}+V t$, for fixed $x_{1}$ and $t \rightarrow \infty$, has the form

$$
Z\left(x_{1}+V t, t\right) \sim C T\left(k_{1}, \omega_{1}\right)=\boldsymbol{Q}\left(x_{1}+V t, \omega_{1}+k_{1} V\right) P\left(k_{1}, \omega_{1}+k_{1} V\right) \mathrm{e}^{\mathrm{i} k_{1} x_{1}} t^{s-1} \mathrm{e}^{-\mathrm{i} \omega_{1} t}
$$

(see BB, equation (4.14)). Here $\left(k_{1}, \omega_{1}\right)$ is a multiple root in $k$ of the dispersion relation $D(k, \omega)=0$ that satisfies a collision criterion, $\operatorname{Im}\left(\omega_{1}\right)=\max$ and $s$ is a rational number that depends on the multiplicity of the colliding roots at $k=k_{1}$, cf. Brevdo (1988). For two simple colliding $k$ roots, $s=\frac{1}{2}$. Therefore, the temporal

Proc. R. Soc. Lond. A (1997) 
growth rate of this contribution depends only on the global normal modes, i.e. on $\omega_{1}$ and $s$, since $\boldsymbol{Q}\left(x_{1}+V t, \omega_{1}+k_{1} V\right)$ is uniformly bounded in time because $\boldsymbol{Q}(x, \omega)$ is $L$ periodic and analytic in $x$, for real $x$ and every fixed $\omega \in \mathbb{C}$. Due to the analyticity and because of (3.4), (3.5), for fixed $k_{1}, \omega_{1}$ and $V$, the function $\boldsymbol{Q}\left(x_{1}+V t, \omega_{1}+k_{1} V\right)$ is not identically zero, for $t>0$, and it can be zero for at most a finite number of points $x_{1}+V t=x_{m}, m=2,3, \ldots, M$, in the period interval $[0, L]$. This shows that, although the asymptotics along the ray $x=x_{1}+V t$ are on the whole determined by the global normal modes, their amplitudes are influenced by the $x$ variability of $\boldsymbol{Q}(x, \omega)$. Since $\boldsymbol{Q}(x, \omega)$ is periodic in $x$, it is tempting to regard its $x$ dependence as benign. However, a smooth periodic function can be highly irregular; for example, integrable but with localized spikes (see also comments in $\S 5$ ).

These considerations underline two important aspects of the wave evolution in unstable spatially periodic flows. First, they show that the growth rates of linear perturbations in such flows are governed by the properties of the global normal modes (3.6), (3.7) of the basic state regardless of the spatial rate of change and the period size of the coefficients of the matrix $\boldsymbol{A}(x)$ in the equation in (3.1). Slow spatial variability of these coefficients, however negligible it may be with respect to other physical parameters, provides by itself no a priori justification for the applicability of the parallel flow assumption. Second, the model dependent spatial variability of solution (3.2) and asymptotics (3.8) is inherently present. It is represented by the $x$ variability of the function $\boldsymbol{Q}(x, \omega)$ and expressed in spatially and time dependent bounded amplitudes multiplying the growing contributions of the global normal modes.

\section{The complex Ginzburg-Landau equation}

The complex Ginzburg-Landau equation (CGL) is a model equation for a class of near critical hydrodynamic stability problems. A history with references of the derivation of the CGL can be found in Stuart \& DiPrima (1978). By scaling the coefficients and restricting consideration to the supercritical case, the CGL can be written in the form

$$
\tilde{\Psi}_{t}=\tilde{\Psi}+(1+\mathrm{i} a) \tilde{\Psi}_{x x}+(-1+\mathrm{i} b)|\tilde{\Psi}|^{2} \tilde{\Psi}
$$

where $a$ and $b$ are arbitrary real numbers. There exists a finite-amplitude wave state of (4.1) of the form

$$
\tilde{\Psi}_{0}(x, t)=\xi \mathrm{e}^{\mathrm{i} \theta}, \quad \xi \in \mathbb{C}, \quad \theta=\alpha x-\beta t .
$$

The parameters of the exact solution (4.2) of (4.1) satisfy

$$
\beta=-b+(a+b) \alpha^{2} \quad \text { and } \quad \rho^{2}+\alpha^{2}=1, \quad \text { with } \rho^{2}=|\xi|^{2} .
$$

A treatment of the normal mode instabilities of the periodic solutions of the CGL equation is given in Stuart \& DiPrima (1978). A consideration of the initial-value problem for (4.1), linearized about the exact state (4.2), and an analysis of the unstable wave packets are presented in BB. There, it is shown that there is a transition, in the parameter space, from absolutely unstable to convectively unstable periodic solutions of the CGL equation. In the present argument we will use both the normal mode theory and the wave packet analysis to analyse the distinction between the local and global instabilities.

Proc. R. Soc. Lond. A (1997) 
Solution (4.2) is periodic in space and oscillatory in time. In the frame of reference moving at speed $c=\beta / \alpha$, with respect to the stationary frame of reference, the solution is periodic in space and stationary. In the moving frame of reference, equation (4.1) reads

$$
\Psi_{t}=\Psi+\left[-\frac{b}{\alpha}+(a+b) \alpha\right] \Psi_{x}+(1+\mathrm{i} a) \Psi_{x x}+(-1+\mathrm{i} b)|\Psi|^{2} \Psi
$$

and the basic state (4.2) is

$$
\Psi_{0}(x)=\xi \mathrm{e}^{\mathrm{i} \alpha x} .
$$

It is important to emphasize that solution $(4.2 a)$ is exact and is, therefore, a uniformly valid solution of equation $(4.1 a)$ in the limit $\alpha \rightarrow 0^{+}$. In order to demonstrate an inconsistency of the parallel flow assumption, we will compare the global stability properties of solution $(4.2 a)$ with those of $\Psi_{0}\left(x_{0}\right)=\xi \mathrm{e}^{\mathrm{i} \alpha x_{0}}$, for a fixed $x_{0}$. This will be done for several cases including the limiting case $\alpha \rightarrow 0^{+}$. We will give an example in which, for $\alpha \rightarrow 0^{+}$, there exists an essential discrepancy between these properties, whereas the fundamental premise of the parallel flow assumption for $(4.2 a)$ is fulfilled to within an arbitrary accuracy. Since the basic state $\Psi_{0}(x)$ given in $(4.2 a)$ is stationary, its temporal variation scale $T_{0}$ is infinite. Therefore, no matter how slow a given normal mode, with a non-zero real part $\omega_{\mathrm{r}}$ of the frequency $\omega$, varies in time, for small $\alpha$, its finite temporal variation scale $\varphi_{0}=2 \pi /\left|\omega_{\mathrm{r}}\right|$ always satisfies the inequality $\varphi_{0} \ll T_{0}$. Hence, in the limit, $\alpha \rightarrow 0^{+}$, the inequality $\lambda_{0} \ll L_{0}$ alone forms a fundamental premise of the parallel flow assumption for perturbations of the stationary basic state $(4.2 a)$ whose characteristic temporal variation scale is finite in this limit.

The results of the comparison of the global properties of the solution $(4.2 a)$ with those of $\Psi_{0}\left(x_{0}\right)=\xi \mathrm{e}^{\mathrm{i} \alpha x_{0}}$ will be reformulated for the stationary frame of reference moving at speed $-c=-\beta / \alpha$ with respect to the frame of $(4.1 a),(4.2 a)$. In the stationary frame of reference, the frequencies of both states (the global and the local) will be altered by the same amount $k c: \omega_{\mathrm{st}}(k)=\omega(k)+k c$, where $k$ is a wave number, $\omega(k)$ is a frequency in the moving frame of reference and $\omega_{\mathrm{st}}(k)$ is the frequency in the absolute stationary frame of reference. Therefore, in the stationary frame of reference, the unstable wave numbers and the growth rates of the normal modes are unchanged, whereas the propagation velocities of the wave packets are equal to those in the moving frame of reference increased by the quantity $c$, for both states. Hence, a contradiction of the results reformulated in the absolute frame of reference will imply a contradiction between the global and the local analysis of $(4.1 a),(4.2 a)$.

The normal mode linear stability theory proceeds by linearising $(4.1 a)$ about the state $(4.2 a)$ with

$$
\Psi(x, t)=\Psi_{0}(x)+\mathrm{e}^{\mathrm{i} \alpha x}\left[A \mathrm{e}^{\mathrm{i}(k x-\omega t)}+B \mathrm{e}^{-\mathrm{i}\left(k^{*} x-\omega^{*} t\right)}\right] .
$$

As a result, one obtains the dispersion relation

$$
D(k, \omega) \equiv\left(\omega+\frac{\beta}{\alpha} k\right)^{2}-2 \mathrm{i} p(k)\left(\omega+\frac{\beta}{\alpha} k\right)-q(k)=0,
$$

where

$$
\begin{aligned}
& p(k)=-k^{2}-2 \mathrm{i} a \alpha k-\rho^{2}, \\
& q(k)=\left(1+a^{2}\right)\left(k^{2}-4 \alpha^{2}\right) k^{2}-2 \rho^{2}(a b-1) k^{2}+4 \mathrm{i} \alpha(a+b) \rho^{2} k .
\end{aligned}
$$

Proc. R. Soc. Lond. A (1997) 


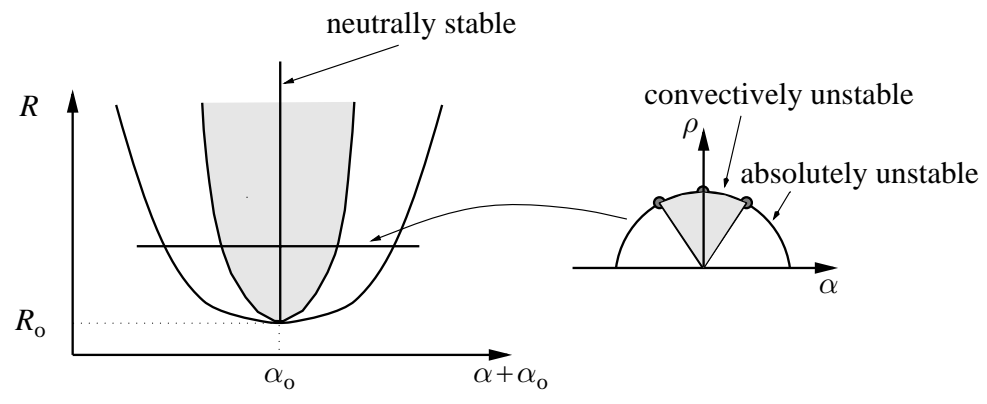

Figure 1. Schematic of the regions of instability obtained using the theory of BB along the branch of periodic states, when $a b=1$.

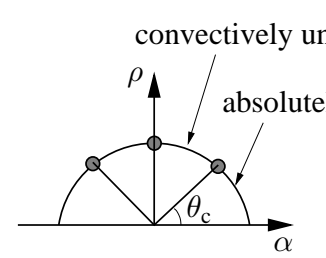

(a)

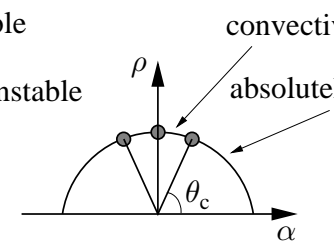

(b)

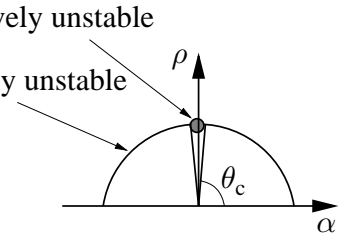

(c)

Figure 2. Exact instability results along the branch of periodic states when $a b=1$. In the limit as $a \rightarrow 0$, the complete branch is absolutely unstable. The parameters are $(a) a=10, \theta_{\mathrm{c}} \approx 45^{\circ}$, (b) $a=1, \theta_{\mathrm{c}} \approx 60^{\circ}$, (c) $a=0.1, \theta_{\mathrm{c}} \approx 86^{\circ}$. The transition from absolute to convective instability occurs when $\theta$ increases through $\theta=\theta_{\mathrm{c}}$, where $\alpha=\cos \theta$.

Note that the dispersion relation is exact and independent of $x$, i.e. it is global, even though the basic state is spatially periodic. When $a b=1$, the complete branch $\rho^{2}+\alpha^{2}=1,0 \leqslant \rho \leqslant 1$, is unstable with the exception of the point $\rho=1$, i.e. $\alpha=0$, for which the solution is neutrally stable. The theory in BB can be applied to show that the region of unstable periodic solutions is composed of two distinct parts. A schematic of the stability properties in the absolute stationary frame of reference is shown in figure 1 . In this figure, $R$ is a control parameter, e.g. Reynolds number, and $R_{0}$ is its critical value, i.e. the one for which a transition from linear stability to instability in a homogeneous flow occurs. In figures $2 a-c$, the effect of the parameter $a$ (with $a b=1$ ) is shown in the same frame. Qualitatively, the picture is the same for all $a, b$ on the curve $a b=1$ : the solution with $\alpha=0$ is neutrally stable and the linearly unstable region $0<\alpha<1$ has two components. There is an $a$-dependent neighbourhood of $\alpha=0$ where the periodic state is convectively unstable and outside this neighbourhood it is absolutely unstable. However, note that in the limit as $a \rightarrow 0$ (with $a b=1$ ) the region of convective instability shrinks to a point. The point where the contributing saddle point crosses the real $\omega$ axis corresponds to the change from absolute to convective instability (cf. BB).

The above results are exact and are a consequence of the Floquet decomposition. The Floquet decomposition can, in this case, be exactly constructed (cf. BB) resulting in the exact dispersion relation. Now we compare the above exact results with an analysis of the linear stability of the basic periodic stationary state $((4.2 a),(4.3))$ using the quasi-homogeneous hypothesis. Let $x_{0}$ be any fixed value of $x$. For this value, we consider the linear stability of the basic state $\Psi_{0}\left(x_{0}\right)$. When $\alpha=0$, the basic state $(4.2 a)$ is parallel and independent of $x$. Moreover, when $\alpha$ is small the basic state varies weakly with $x$ and, therefore, it would appear to be reasonable to suppose that the quasi-homogeneous hypothesis for $|\alpha| \ll 1$ would be valid.

Proc. R. Soc. Lond. A (1997) 
Let $\Psi(x, t)=\Psi_{0}(x)+U(x, t)$. In the parallel stability analysis, we linearize $(4.1 a)$ about the state $\Psi_{0}(x)$ and then freeze $x$ at a constant $x_{0}$ where it appears in the coefficients to obtain

$$
U_{t}=U+\left[-\frac{b}{\alpha}+(a+b) \alpha\right] U_{x}+(1+\mathrm{i} a) U_{x x}+(-1+\mathrm{i} b)\left\{2 \rho^{2} U+\left[\Psi_{0}\left(x_{0}\right)\right]^{2} U^{*}\right\} .
$$

Normal mode substitution of the form

$$
U(x, t)=A \mathrm{e}^{\mathrm{i}(k x-\omega t)}+B \mathrm{e}^{-\mathrm{i}\left(k^{*} x-\omega^{*} t\right)}
$$

into equation (4.7) leads, in the case $a b=1$, to the dispersion relation

$$
D_{\mathrm{P}}(k, \omega) \equiv\left(\omega+\frac{\beta}{\alpha} k\right)^{2}-2 \mathrm{i} s(k)\left(\omega+\frac{\beta}{\alpha} k\right)-r(k)=0,
$$

where the subscript $\mathrm{P}$ indicates the 'parallel' dispersion relation and

$$
\begin{aligned}
& s(k)=-k^{2}-\rho^{2}+\alpha^{2} \\
& r(k)=\left(1+a^{2}\right) k^{4}-2 k^{2}+\alpha^{4}-2 \rho^{2} \alpha^{2}+3 \rho^{4} / a^{2} .
\end{aligned}
$$

The parallel (or local) dispersion relation (4.9) predicts dramatically different results from those given by the exact dispersion relation (4.5). Let us consider the values of the stability exponents predicted by the dispersion relation $D_{\mathrm{P}}(k, \omega)=0$. For example, let us take $k=0$, which is the simplest homogeneous class of perturbations. Then $D_{\mathrm{P}}(0, \omega)=0$ gives

$$
\left.\omega_{\mathrm{P}}(k)\right|_{k=0}=\mathrm{i}\left(2 \alpha^{2}-1\right) \pm \rho^{2}\left(\frac{3}{a^{2}}-1\right)^{1 / 2} .
$$

Therefore, for $a^{2}<3$, the parallel analysis, in a class of perturbations with $k=0$, predicts that the basic state is stable for $0<\alpha^{2}<\frac{1}{2}$, whereas the exact analysis gives neutral stability for $\alpha=0$ and instability for $0<\alpha^{2}<\frac{1}{2}$.

Now, consider the general case with $k$ non-zero. When $k$ is real and non-zero, $D_{\mathrm{P}}(k, \omega)=0$ results in

$$
\omega_{\mathrm{P}}(k)=-\frac{\beta}{\alpha} k+\mathrm{i} s(k) \pm\left\{r(k)-[s(k)]^{2}\right\}^{1 / 2}=-\frac{\beta}{\alpha} k+\mathrm{i}\left(2 \alpha^{2}-1-k^{2}\right) \pm \sqrt{\delta(k)},
$$

where

$$
\delta(k)=a^{2} k^{4}-4 \rho^{2} k^{2}+\left(\frac{3}{a^{2}}-1\right) \rho^{4} .
$$

With some straightforward algebra one can deduce that the imaginary part of $\omega_{\mathrm{P}}(k)$, given by (4.12), for real $k$, is negative for $\alpha^{2}<\min \left\{1 /\left(a^{2}+1\right), \frac{1}{2}\right\}$. This is the range of $\alpha$ along the branch where the parallel analysis predicts stability for any $a \in \mathbb{R}$, with $a b=1$. In this range the exact analysis gives instability which by itself is a clear contradiction. However, a consideration of the wave packets shows that there is a more important distinction between the local and global predictions.

For the particular case $a=1$, the parallel stability range is $0<\alpha<1 / \sqrt{ } 2$. However, the periodic state treated globally is convectively unstable for $0<\alpha<0.479$ and absolutely unstable for $0.479<\alpha<1$. Consequently, in the region $0.479<\alpha<$ $1 / \sqrt{ } 2$, the flow treated under the quasi-homogeneous hypothesis is stable, whereas in reality the periodic flow is absolutely unstable (see figure 3 for the stability regions in the absolute frame of reference). In the overlapping region, the spatial variability

Proc. R. Soc. Lond. A (1997) 


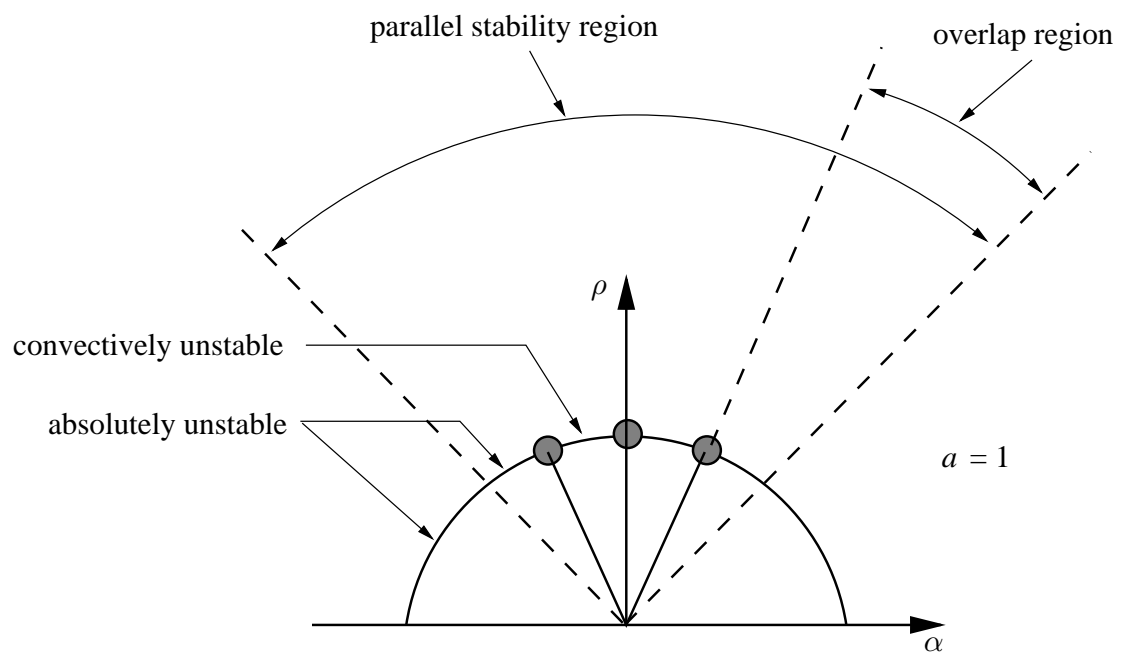

Figure 3. Stability region predicted by the parallel stability theory, and the exact results, for $a=1$. In the overlap region, where the flow is absolutely unstable, the parallel theory predicts stability.

is moderate. From the physical point of view the local and global predictions are strongly contradictory: a spatially moderately varying solution of the equations of motion, for which the parallel flow assumption predicts stability, may not even represent a physical end state at any location in space, since in reality it is globally absolutely unstable.

The case $a=2$ presents a direct example of the quantitative inconsistency. For $0 \leqslant \alpha^{2}<\frac{1}{5}$, the growth rate $\operatorname{Im}\left(\omega_{\mathrm{P}}(k)\right), k \in \mathbb{R}$, predicted by the parallel analysis, equation (4.12), reaches its maximum of

$$
\max _{k \in \mathbb{R}} \operatorname{Im}\left[\omega_{\mathrm{P}}(k)\right]=\operatorname{Im}\left(\omega_{0}\right)=\left.\frac{\alpha^{2}\left(a^{2}+1\right)-1}{a^{2}}\right|_{a=2}=\frac{1}{4}\left(5 \alpha^{2}-1\right)
$$

at

$$
k=k_{0}=\left.\frac{\sqrt{1-\alpha^{2}}}{a}\right|_{a=2}=\frac{1}{2} \sqrt{1-\alpha^{2}} .
$$

Consequently, in the limit $\alpha \rightarrow 0^{+}$, the least stable wave number $k_{0}=\frac{1}{2}\left(\sqrt{1-\alpha^{2}}\right)$ tends to $\frac{1}{2}$, it remains bounded from below away from zero. The flow $(4.2 a)$, treated as locally parallel, is uniformly stable in this limit because the growth rates $\operatorname{Im}\left[\omega_{\mathrm{P}}(k)\right] \leqslant$ $\operatorname{Im}\left(\omega_{0}\right), k \in \mathbb{R}$, remain negative and uniformly bounded from above away from zero. For instance, for $0<\alpha<\frac{1}{5}$, they are uniformly bounded by $-\frac{1}{5}$. The real part of the frequency $\operatorname{Re}\left[\omega_{\mathrm{P}}(k)\right]$ for the flow $(4.2 a)$ in the parallel analysis behaves, to the leading order in $\alpha^{-1}$, as $\operatorname{Re}\left[\omega_{\mathrm{P}}(k)\right] \asymp-k \beta / \alpha \asymp-k b / \alpha$, in the limit $\alpha \rightarrow 0^{+}$(see (4.12), (4.3)). The temporal variation scale of the most unstable local normal mode $\varphi_{0}=$ $2 \pi /\left|\operatorname{Re}\left[\omega_{\mathrm{P}}\left(k_{0}\right)\right]\right| \approx 2 \pi \alpha /\left(k_{0} b\right)=4 \pi \alpha /\left(b \sqrt{1-\alpha^{2}}\right)$ is diminishing in this limit, so the inequality $\varphi_{0} \ll T_{0}$ is satisfied uniformly in $\alpha$, for small $\alpha$, where, we recall, $T_{0}$ is the infinite temporal variation scale of the stationary solution $(4.2 a)$. On the other hand, the exact periodic basic state $(4.2 a)$ is unstable for $0<\alpha<1$ and neutrally stable for $\alpha=0$. From the point of view of an experimentalist observing the stationary flow (4.2 a) locally, this flow, for $\alpha \rightarrow 0^{+}$, is arbitrarily slowly varying, since its spatial variation scale $L_{0}=2 \pi / \alpha$ becomes unbounded in this limit. A theoretician treating

Proc. R. Soc. Lond. A (1997) 
this flow by applying the parallel flow analysis to equation (4.1 $a$ ) will establish that, for $\alpha \rightarrow 0^{+}$, the formal criterion for a slow variability of the flow is fulfilled to within an arbitrary accuracy. Indeed, in this limit, the characteristic wavelength $\lambda_{0}=2 \pi / k_{0}=4 \pi / \sqrt{1-\alpha^{2}}$ of the least stable waves is approximately $4 \pi$. So the ratio $\lambda_{0} / L_{0}=\alpha / k_{0}=2 \alpha / \sqrt{1-\alpha^{2}}$ becomes arbitrarily small, for $\alpha \rightarrow 0^{+}$.

The last case shows that, no matter how high the degree of accuracy, to which the fundamental premise of the quasi-homogeneous hypothesis is fulfilled, the parallel flow analysis applied to solution $(4.2 a)$ of equation $(4.1 a)$ may produce stability results that directly contradict the exact stability results.

The inequality (2.1) is violated in the last example for any $0<\alpha<1 / \sqrt{ } 5$. As pointed out in $\S 2$, the explanation for this is that the quasi-periodic global normal modes (3.6) are associated with the continuous spectrum of the corresponding spectral problem that is analogous to the spectral problem (2.12). In the present example, it is a generalized spectral problem obtained by making a global mode ansatz

$$
Z(x, t)=\hat{Z}(x, \omega) \mathrm{e}^{-\mathrm{i} \omega t}
$$

in the homogeneous equation associated with the equation in (3.1). This spectral problem reads

$$
\left[-\mathrm{i} \frac{\mathrm{d}}{\mathrm{d} x}+\mathrm{i} \boldsymbol{A}(x)\right] \hat{Z}(x, \omega)=\omega \boldsymbol{M} \hat{Z}(x, \omega), \quad \omega \in \mathbb{C}, \quad-\infty<x<\infty .
$$

The eigenvalue spectrum of the spectral problem $(4.17)$ in $L_{2}(\mathbb{R})$ is empty. Indeed, the general solution of (4.17) is

$$
\hat{Z}(x, \omega)=\boldsymbol{\Phi}(x, \omega) h \equiv \boldsymbol{Q}(x, \omega) \mathrm{e}^{\boldsymbol{B}(\omega) x} h,
$$

where $\boldsymbol{\Phi}(x, \omega)$ is defined in (3.4) and $h \in \mathbb{C}$ is a constant vector (see $\S 3$ ). For any $\omega \in \mathbb{C}$, for which there exists a real $k$ such that the pair $(k, \omega)$ satisfies the dispersion relation (3.7), there exists a solution of (4.17) bounded for all $x \in \mathbb{R}$, namely

$$
\hat{Z}(x, \omega)=\boldsymbol{Q}(x, \omega) \mathrm{e}^{\mathrm{i} k x} h .
$$

This can be deduced by substituting (4.19) into (4.17) and making use of (3.4) and (3.5). With bounded $\hat{Z}(x, \omega)$ given in (4.19), the global mode (4.16) is precisely the global normal mode (3.6). However, for each $\omega \in \mathbb{C}$ and $h \in \mathbb{C}^{N}$, the $\mathbb{C}^{N}$ vector norm of a non-trivial solution (4.18) of (4.17) cannot decay to zero for both $x \rightarrow \infty$ and $x \rightarrow-\infty$. Consequently, any such solution is not square integrable on $\mathbb{R}$ and, thus, the eigenvalue spectrum of $(4.17)$ is empty in $L_{2}(\mathbb{R})$. Therefore, a definition of the global modes (4.16) for treating global stability that requires square integrable eigenfunctions $\hat{Z}(x, \omega)$ (cf. (2.15)) is not applicable to spatially periodic flows, even with slow spatial variability.

\section{Discussion and concluding remarks}

The analysis of spatially periodic flows has highlighted the subtle role of the continuous spectrum when comparing local and global stability exponents and provided a setting for exact definitions of global and local absolute and convective instabilities. Although the precise arguments have been presented for spatially periodic flows, predominantly, some general conclusions can be drawn and the ideas carried over in an appropriate way, with some degree of plausibility, to certain non-periodic flows.

Proc. R. Soc. Lond. A (1997) 

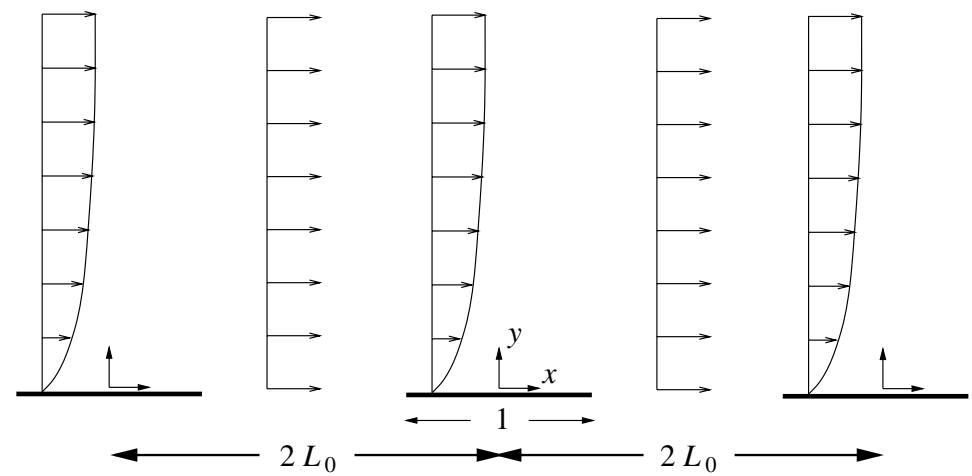

Figure 4. Schematic of a periodic extension of the flow past a finite flat plate. $L_{0} \gg 1$.

Since a continuous spectrum appears in the global spectrum of many spatially varying non-periodic basic states, if a component of the continuous spectrum is unstable then the subtle issues raised here about comparing local and global exponents will be relevant.

Another important issue raised by the analysis of spatially periodic flows is that the concept of local stability for them may not exist. This observation suggests that a similar issue may be relevant for spatially varying but non-periodic flows, particularly when they can be embedded in periodic flows. For example, consider the two-dimensional boundary layer on a plate of finite streamwise extent in a flow which is uniform and parallel to the plate far upstream. Let $x$ be a coordinate along the plate and $y$ perpendicular to the plate in a coordinate system with the origin in the middle of the plate, and let the plate extend from $x=-\frac{1}{2}$ to $x=\frac{1}{2}$ (see figure 4). Since the plate has a finite length, which is essential for our discussion, the horizontal extent of the boundary layer is finite and, moreover, for all physical purposes the flow, far upstream and far downstream from the plate, can be viewed as uniform. This means that starting with some $L_{0}$, with $L_{0} \gg 1$, the flow for $x \leqslant-L_{0}$ and for $x \geqslant L_{0}$ can be assumed to be uniform. Now, let us consider the flow for $-L_{0} \leqslant x \leqslant L_{0}, 0<y<\infty$. For $x= \pm L_{0}$ it is the same uniform flow. Therefore, we propose to extend it periodically in $x$ from $x \in\left[-L_{0}, L_{0}\right]$ into the entire infinite domain $x \in \mathbb{R}, 0<y<\infty$. Physically, this periodic continuation may be viewed as an unbounded plane flow, with a countable set of plates of length one placed with their centres at the points $x_{m}^{\mathrm{c}}=2 m L_{0}, m=0, \pm 1, \pm 2, \ldots$ (cf. figure 4 ).

The linear perturbation dynamics of the resulting $x$-periodic flow are, according to the theory in BB, global. The use of the term global here is not to be confused with absolute instability; it implies that all $x$ stations are globally intertwined and $x$ variability is determined by a globally defined eigenfunction. The stability treatment is as outlined in $\S 3$, with $L=2 L_{0}$, and with the initial condition in the initial-value problem (3.4) formulated at $x=-L_{0}$, i.e. $\boldsymbol{\Phi}\left(-L_{0}, \omega\right)=\boldsymbol{I}_{N}$. Consequently, in the range of Reynolds numbers where, according to the experimental observations, a linear stability treatment may apply, the boundary layer on a plate of finite streamwise extent can have only global $x$-independent stability properties. This conclusion may sound rather surprising, particularly in view of the experimental evidence concerning the convective nature of the spatial evolution of unstable linear wave packets in the boundary layer on a flat plate (cf. Gaster 1975; Gaster \& Grant 1975), which is supported by the theoretical stability results obtained in the framework of the parallel flow analysis (cf. Brevdo 1995). One possibility to resolve this difficulty is

Proc. R. Soc. Lond. A (1997) 
to conclude that the periodic continuation of the flow, or the existence of the flow with an infinite periodic sequence of plates far away from one another and a uniform velocity half way between the plates, is unjustified.

Another possible explanation is as follows. Suppose that the periodic continuation is valid and the linear dynamics of a boundary layer of finite streamwise extent are essentially global. The experimentally observed emergence of linear growing waves that starts at a distinct distance $x_{n}$ from the leading edge, rather than globally on the entire plate, can be explained in the framework of the periodic flow by the $x$ variability of the function $\boldsymbol{Q}(x, \omega)$ in the expression (3.2) for the solution of the initialvalue problem in the global approach. Although no supporting numerical evidence for this assertion is available at the present time, this possibility is inherently present in the form of the solution. Referring to the form of the solution in $\S 3$, the global spatially independent normal modes in the periodically continued flow are unstable for a certain range of wave numbers, but the amplitudes of the linear wave packets depend on $\boldsymbol{Q}\left(x_{1}+V t, \omega_{1}+k_{1} V\right)$ (see (3.8)). Furthermore, it is the strong spatial variability of the function $\boldsymbol{Q}(x, \omega)$ combined with the convective nature of instability of the periodic flow that may cause a gradual spatial evolution of infinitesimal wave packets in the boundary layer.

Based on the above remarks, a possible scenario for the linear destabilization of the boundary layer on a plate of finite streamwise extent, when the flow far upstream is laminar, can be outlined within the global context as follows. There exists a critical Reynolds number $R_{\mathrm{c}}$ for which the periodically extended flow described above becomes globally linearly unstable. For purposes of discussion we can assume, based only on the experimental observations, that, for supercritical Reynolds numbers $R>R_{\mathrm{c}}$, the periodically extended flow is absolutely stable, but convectively unstable. So the disturbances emerging everywhere on the plate and, in particular, at its leading edge travel downstream, while the time dependent part of the corresponding asymptotics (3.8) grows. Since this growth takes place during the propagation in space, the asymptotic, as well as actual, amplitudes of the wave packets are influenced by the spatial variability of the function $\boldsymbol{Q}(x, \omega)$, as is seen from (3.8) and (3.2). Referring to the asymptotics (3.8), if, for all unstable rays $x=x_{1}+V t$, the absolute values of the entries of the matrix $\boldsymbol{Q}\left(x, \omega_{1}+k_{1} V\right)$ are sufficiently small for the values of $x$ between the leading edge value and some value $x_{n}$ downstream on the plate, which we would argue is plausible, no disturbances would be detectable in the experiments for $x<x_{n}$ because the amplitudes in this portion of the flow are too small. Then the point $x_{n}$ would look like a neutral stability point. In other words, the foremost point $x_{n}$ at which the growing disturbances first appear in the experiments is not necessarily the one where the local modes become unstable. In any case, such local modes in the periodic setting cannot be consistently defined. In the global context, $x_{n}$ is the point at which the combined effect - of the growth of the propagating wave packets, governed by the global dispersion relation, and of the spatial variability of the amplitudes, because of $\boldsymbol{Q}(x, \omega)$-results in the appearance of measurable amplitudes.

For experiments with ribbon induced oscillatory disturbances, with the $x$ variability modelled as periodic variation, the spatial asymptotics of the response, for $x \rightarrow \infty$, under the hypotheses of the current discussion, would be given by

$$
Z(x, t) \sim C S\left(k_{1}, \omega_{1}\right)=\boldsymbol{Q}\left(x, \omega_{1}\right) P\left(k_{1}, \omega_{1}, x_{1}\right) \mathrm{e}^{-\mathrm{i} \omega_{1} t} \mathrm{e}^{-\operatorname{Im}\left(k_{1}\right) x} \mathrm{e}^{\mathrm{i} \operatorname{Re}\left(k_{1}\right) x}
$$

(see BB, equation (5.8)), where again the influence of the spatial variability of $\boldsymbol{Q}\left(x, \omega_{1}\right)$ on the asymptotics is evident.

Proc. R. Soc. Lond. A (1997) 
Similar considerations can be, in principle, applied to any stationary spatially varying flow or medium (not necessarily slowly varying) that can be modelled as periodic in the homogeneous spatial direction. The assumption that a slowly spatially developing flow at a location $x_{0}$ experiences only the influence of its close neighbourhood and can be, therefore, treated as locally parallel generally disregards the basic fact that this fictitious local flow may be simply an artificial section of the real dynamically globally intertwined flow. A justification of this assumption in realistic particular cases, in which it might be for whatever reason applicable, may be not less formidable than a treatment of the global stability of the flow. This may also point to the necessity to reappraise the applicability of a WKBJ analysis for studying instabilities in slowly spatially varying flows that can be modelled as spatially periodic. As a matter of fact, in a WKBJ analysis it is explicitly assumed that the wave number and frequency are functions of $x$ and this assumption contradicts the concept of the instability for any spatially developing flow that can be modelled as periodic. Thus, it is reasonable to conjecture that the instability for such flows is only globally consistently defined.

As in the beginning of this section, we would like to point out here as well that the flow with a periodic array of plates and the periodic embedding of flows are of a suggestive, hypothetical nature. The consideration, however, emphasizes and highlights the importance of the global viewpoint and will hopefully stimulate further interest and discussion in this direction.

On the other hand, even without referring to the flow with a periodic array of plates, we can think about a vector function giving a velocity-pressure field $(U, P)$ for a flow around only one plate in a domain that extends in the horizontal direction from $-L_{0}$ to $L_{0}$, whereas this field is for all physical purposes homogeneous at $x= \pm L_{0}$. The flow at $x= \pm L_{0}$ is parallel to the plate. If the local stability analysis is applicable for obtaining an estimate of the global stability properties of this slowly spatially varying vector field $(U, P)$ then it should be applicable in the same way to the vector field obtained by a periodic continuation of the field $(U, P)$ regardless of whether this periodic field represents any flow or not. In other words, if the linear stability PDE for the original field can be treated by using the local approach then the PDE, with periodically extended coefficients of the original PDE, can also be treated in the same way. However, for the PDE with periodically extended coefficients, the asymptotics of the solution are only globally consistently defined, according to the theory in $\S \S 2$ and 3 .

In a recent work, Hunt (1995) has constructed an example in which an $x$-dependent basic state is locally absolutely unstable everywhere but globally stable. The example is based on a complex Ginzburg-Landau equation with coefficients depending linearly on $x$. Although the example considered by Hunt is not an obviously physically realizable flow, the fact that the global behaviour of the solutions of the PDE, with spatially slowly varying coefficients, is qualitatively in contradiction to the parallel local behaviour provides support to the underlying result of the present paper.

In conclusion, we remark that in a recent work of Brevdo \& Bridges (1997), the framework of global instabilities has been extended to the case of time-periodic basic states. In this paper, a Floquet decomposition in time is used to give an exact characterization of global absolute and convective instabilities for temporally oscillating flows. From the analysis of the paper it follows that, for this class of flows as well, only global linear perturbation dynamics are consistently defined.

This research was conducted at the Mathematical Research Institute in Oberwolfach under the

Proc. R. Soc. Lond. A (1997) 
auspices of the Research in Pairs Program which is sponsored by the Volkswagen Foundation. The authors are grateful for the delightful and stimulating environment provided by the Institute. The support of the Sonderforschungsbereich 404 'Multi-field problems in the continuum mechanics' at the University of Stuttgart is gratefully acknowledged. We also thank Robert Hunt for providing us with a copy of his D.Phil. dissertation.

\section{References}

Acheson, D. J. 1993 A pendulum theorem. Proc. R. Soc. Lond. A 443, 239-245.

Acheson, D. J. \& Mullin, T. 1993 On upside-down pendulums. Nature 366, 215-216.

Brevdo, L. 1988 A study of absolute and convective instabilities with an application to the Eady model. Geophys. Astrophys. Fluid Dyn. 40, 1-92.

Brevdo, L. 1995 Convectively unstable wave packets in the Blasius boundary layer. Z. Angew. Math. Mech. 75, 423-436.

Brevdo, L. \& Bridges, T. J. 1996 Absolute and convective instabilities of spatially periodic flows. Phil. Trans. R. Soc. Lond. A 354, 1027-1064.

Brevdo, L. \& Bridges, T. J. 1997 Absolute and convective instabilities of temporally oscillating flows. Z. Angew. Math. Phys. 48, 290-309.

Chomaz, J.-M., Huerre, P. \& Redekopp, L. G. 1991 A frequency selection criterion in spatially developing flows. Stud. Appl. Math. 84, 119-44.

Drazin, P. G. \& Johnson, R. S. 1989 Solitons: an introduction. Cambridge University Press.

Drazin, P. G. \& Reid, W. H. 1981 Hydrodynamic stability. Cambridge University Press.

Dunford, N. \& Schwartz, J. T. 1963 Linear operators, vol. 2. New York: Wiley-Interscience.

Eastham, M. S. P. 1973 The spectral theory of periodic differential equations. Scottish Academic Press.

Gaster, M. 1975 A theoretical model of a wave packet in the boundary layer on a flat plate. Proc. R. Soc. Lond. A 347, 271-289.

Gaster, M. \& Grant, I. 1975 An experimental investigation of the formation of a wave packet in a laminar boundary layer. Proc. R. Soc. Lond. A 347, 253-269.

Hall, P. 1978 The linear stability of flat Stokes layers. Proc. R. Soc. Lond. A 359, 151-166.

Hall, P. \& Smith, F. T. 1991 On strongly nonlinear vortex/wave interactions in boundary-layer transition. J. Fluid Mech. 227, 641-666.

Huerre, P. \& Monkewitz, P. A. 1990 Local and global instabilities in spatially developing flows. A. Rev. Fluid Mech. 22, 473-537.

Hunt, R. E. 1995 Spatially developing flows with localized forcing. D.Phil. dissertation, DAMTP, Cambridge University.

Kerczek, C. von \& Davis, S. H. 1974 Linear stability theory of oscillatory Stokes layers. J. Fluid Mech. 62, 753-773.

Kreiss, H.-O. \& Lorenz, J. 1989 Initial-boundary value problems and the Navier-Stokes equations. Academic.

Kuchment, P. 1993 Floquet theory for partial differential equations. Basel: Birkhäuser.

Levi, M. \& Weckesser, W. 1995 Stabilization of the inverted pendulum by high frequency vibrations. SIAM Rev. 37, 219-223.

Serrin, J. 1959 On the stability of viscous fluid motions. Arch. Ration Mech. Analysis 3, 1-13.

Smith, F. T. 1979 On the non-parallel flow stability of the Blasius boundary layer. Proc. R. Soc. Lond. A 366, 91-109.

Stewart, P. A. \& Smith, F. T. 1987 Three-dimensional instabilities in steady and unsteady non-parallel boundary layers, including effects of Tollmien-Schlichting disturbances and cross flow. Proc. R. Soc. Lond. A 409, 229-248.

Stuart, J. T. \& DiPrima, R. C. 1978 The Eckhaus and Benjamin-Feir resonance mechanisms. Proc. R. Soc. Lond. A 362, 27-41. 